\title{
On Global Optimization for Informative Path Planning
}

Per Boström-Rost, Daniel Axehill and Gustaf Hendeby

The self-archived postprint version of this journal article is available at Linköping University Institutional Repository (DiVA):

http:// urn.kb.se/ resolve?urn=urn:nbn:se:liu:diva-151461

N.B.: When citing this work, cite the original publication.

Boström-Rost, P., Axehill, D., Hendeby, G., (2018), On Global Optimization for Informative Path Planning, IEEE Control Systems Letters, 2(4), 833-838.

https:// doi.org/ 10.1109/ LCSYS.2018.2849559

Original publication available at:

https:// doi.org/ 10.1109/ LCSYS.2018.2849559

Copyright: Institute of Electrical and Electronics Engineers (IEEE)

http:// www.ieee.org/ index.html

(C)2018 IEEE. Personal use of this material is permitted. However, permission to reprint/republish this material for advertising or promotional purposes or for creating new collective works for resale or redistribution to servers or lists, or to reuse any copyrighted component of this work in other works must be obtained from the IEEE. 


\title{
On Global Optimization for Informative Path Planning
}

\author{
Per Boström-Rost, Daniel Axehill, and Gustaf Hendeby
}

\begin{abstract}
The problem of path planning for mobile sensors with the task of target monitoring is considered. A receding horizon optimal control approach based on the information filter is presented, where the limited field of view of the sensor can be modeled by introducing binary variables. The resulting nonlinear mixed integer problem to be solved in each sample, with no apparent tractable solution, is shown to be equivalent to a problem that robustly can be solved to global optimality using off-the-shelf optimization tools.
\end{abstract}

\section{INTRODUCTION}

This paper addresses the problem of finding a path for a mobile sensor in a bounded environment such that the sensor collects measurements that maximize the information gain of targets in the area. This problem is known as informative path planning (IPP) [1] and can be considered as a variant of classical motion planning for a certain choice of objective function. In classical motion planning, the objective is typically to find the shortest path between the locations, whereas IPP seeks to maximize the information gain subject to budget constraints that limit the number of measurements that can be collected. Since the increase in knowledge about the targets that is possible to acquire in the future depends on what is already known, IPP can be considered as a feedback loop to the data fusion process and it is thus closely related to the field of sensor management [2].

A simplification of the IPP problem is the problem of sensor scheduling or sensor selection. Instead of planning an informative path for a mobile sensor, the sensors are static and the challenge is to select when to use which sensor. This problem has been studied extensively and several efficient approaches have been proposed [3-5]. Another IPP problem, path planning for target tracking, is studied in [6].

IPP problems are usually posed as optimization problems that in general are nonconvex due to nonlinear sensor models, motion models or information representation, which means that a globally optimal solution typically can not be guaranteed. Locally optimal solutions are however obtainable using numerical optimization [7, 8], sampling-based techniques [9] or information-theoretic approaches [10-12].

This work was partially supported by the Wallenberg AI, Autonomous Systems and Software Program (WASP) funded by the Knut and Alice Wallenberg Foundation.

P. Boström-Rost, D. Axehill, and G. Hendeby are with the Divison of Automatic Control, Linköping University, Sweden \{per.bostrom-rost, daniel.axehill, gustaf .hendeby\}@liu.se
The papers $[5,13,14]$ discuss how the globally optimal sensor trajectory can be computed if the control input is restricted to a discrete set of admissible controls, and also develop approximate algorithms with suboptimality guarantees. The main result of this work is a theorem that can be used to reformulate a class of IPP problems such that globally optimal trajectories are obtained, without restricting the control input to a discrete set.

In this paper, it is assumed that a mobile sensor can obtain linear measurements of targets that are within field of view (FoV) of the sensor, and the IPP problem is formulated as a mixed integer semidefinite program, or to be more specific, a mixed binary semidefinite program. If the computational burden is ignored, the most straightforward approach to compute the optimal solution to a problem of this type is to perform an exhaustive search, in which a convex problem is solved for each possible combination of the binary variables. The global solution is then the one that gives the smallest optimal value. The number of convex problems that need to be solved increases exponentially with the number of binary variables, which means that the cost of performing an exhaustive search quickly becomes overwhelming. This paper uses branch-and-bound $(\mathrm{BnB})[15]$ to avoid enumerating all possible combinations.

$\mathrm{BnB}$ methods maintain lower and upper bounds on the global objective value by solving subproblems of the original problem, usually obtained using convex relaxations. Hence, the proposed algorithm can be terminated at any time with a certificate that limits the level of suboptimality.

The contribution of this paper is an approach to generate globally optimal trajectories for a mobile sensor. We formulate an IPP problem as a receding horizon optimal control problem with a matrix-valued state that represents the acquired information and show that the resulting nonconvex optimization problem can be solved to global optimality, or to a user-defined certified level of suboptimality, using off-the-shelf optimization tools.

\section{Problem Formulation}

This section defines the sensor and target models, and proposes a receding horizon control approach to the informative path planning problem.

\section{A. Motion and measurement models}

Consider a mobile sensor, where the dynamics are governed by the following linear sensor motion model:

$$
x_{k+1}=A_{k} x_{k}+B_{k} u_{k},
$$


where $x_{k}$ is the sensor state and $u_{k}$ is the control input. The set of feasible states is denoted by $\mathcal{X}$ and the set of admissible controls is denoted by $\mathcal{U}$.

The task of the mobile sensor is to monitor properties of a number of targets $i \in\{1, \ldots, M\}$ at known locations $p^{i}$. Each target remains at its static location, but its property of interest is assumed to evolve according to a linear model

$$
z_{k+1}^{i}=F_{k} z_{k}^{i}+G_{k} w_{k}^{i}
$$

where $z_{k}^{i}$ is the state of target property $i$ and $w_{k}^{i}$ is assumed to be a white Gaussian noise process with covariance $\mathbb{E}\left\{w_{k}^{i} w_{k}^{i \mathrm{~T}^{\prime}}\right\}=Q_{k} \succ 0$. The matrix $F_{k}$ is assumed to be nonsingular.

The sensor acquires information about the target properties through imperfect observations modeled by the following sensor measurement model:

$$
y_{k}^{i}=H\left(x_{k}, p^{i}\right) z_{k}^{i}+e_{k}^{i},
$$

where $y_{k}$ is the measurement obtained by the sensor and $e_{k}^{i}$ represents the measurement error, which is assumed to be a white Gaussian noise process with covariance $\mathbb{E}\left\{e_{k}^{i} e_{k}^{i \top}\right\}=R_{k} \succ 0$. It is also assumed that the measurement noise for different target properties are independent. The matrix $H\left(x_{k}, p^{i}\right)$ is a function of the state of the mobile sensor and the location of the target, which can be used to model the fact that the sensor can observe only the targets that are in the vicinity of its position.

The information gain from observations can be represented in various forms. The information filter [16] is suitable when the distribution of the state vector can be approximated with a Gaussian distribution. It makes use of an information matrix $\mathcal{I}$ to represent the accuracy of the state estimate. With the evolution of the target state given by (2) and the sensor measurement model given by (3), the measurement and time update equations of the information matrix in an information filter are given by

$$
\begin{aligned}
\mathcal{I}_{k \mid k} & =\mathcal{I}_{k \mid k-1}+H\left(x_{k}, p^{i}\right)^{\top} R_{k}^{-1} H\left(x_{k}, p^{i}\right), \\
\mathcal{I}_{k+1 \mid k} & =\left(F_{k} \mathcal{I}_{k \mid k}^{-1} F_{k}^{\top}+G_{k} Q_{k} G_{k}^{\top}\right)^{-1} .
\end{aligned}
$$

The interpretation of (4) is that the information gained from a new measurement is captured by the term $H\left(x_{k}, p^{i}\right)^{\top} R_{k}^{-1} H\left(x_{k}, p^{i}\right)$, the information accumulated in all measurements until time $k$ is represented by $\mathcal{I}_{k \mid k}$, and that the time update implies forgetting some of the old information. By denoting $\mathcal{I}_{k}=\mathcal{I}_{k \mid k}$ and $J\left(x_{k}\right)=H\left(x_{k}, p^{i}\right)^{\top} R_{k}^{-1} H\left(x_{k}, p^{i}\right)$, the recursion can be written in a more compact form:

$$
\mathcal{I}_{k+1}=\left(F_{k} \mathcal{I}_{k}^{-1} F_{k}^{\top}+G_{k} Q_{k} G_{k}^{\top}\right)^{-1}+J\left(x_{k+1}\right) .
$$

Note that the information matrix recursion does not depend on the actual measurements. Hence, it is possible to use the information filter in order to estimate the accuracy of future estimates without knowing the measurements.

\section{B. The informative path planning problem}

The objective of the informative path planning problem is to maximize the information acquired by the mobile sensor, reflected by a suitable performance measure. Unlike a classical motion planning problem, the goal is not to find a path to a given goal state but rather to find a path from where the sensor can obtain maximally informative measurements. Various measures could be used, but in general the objective function can be defined as a function of the information matrices, the state of the mobile sensor and the control inputs:

$$
L(\mathcal{I}, x, u)=\sum_{k=0}^{N-1} l_{k}\left(\mathcal{I}_{k}, x_{k}, u_{k}\right)+m\left(\mathcal{I}_{N}, x_{N}\right),
$$

where $l_{k}(\cdot)$ and $m(\cdot)$ are the stage costs and the terminal cost respectively, and $N$ is the planning horizon.

We are now ready to state the problem. A receding horizon approach is employed and a finite horizon optimal control problem that can be formulated as follows is solved repeatedly. Given an initial state $\bar{x}_{0}$ of the mobile sensor and an initial information matrix $\overline{\mathcal{I}}_{0}$, the optimization problem to be solved is in the form

$$
\begin{array}{cl}
\underset{x_{k}, u_{k}, \mathcal{I}_{k}}{\operatorname{minimize}} & \sum_{k=1}^{N-1} l_{k}\left(\mathcal{I}_{k}, x_{k}, u_{k}\right)+m\left(\mathcal{I}_{N}, x_{N}\right) \\
\text { subject to } & x_{k+1}=A_{k} x_{k}+B_{k} u_{k}, \\
& \mathcal{I}_{k+1}=\left(F_{k} \mathcal{I}_{k}^{-1} F_{k}^{\top}+G_{k} Q_{k} G_{k}^{\top}\right)^{-1}+J\left(x_{k+1}\right) \\
& x_{k} \in \mathcal{X}, \quad u_{k} \in \mathcal{U} \\
& x_{0}=\bar{x}_{0}, \quad \mathcal{I}_{0}=\overline{\mathcal{I}}_{0} .
\end{array}
$$

It should be noted that $\mathcal{I}_{k}$ and $x_{k}$ can be computed from $u$. However, as commonly done in model predictive control (MPC), introducing these as auxiliary decision variables connected by constraints provides a more favorable problem structure.

Due to the nonlinear equality constraints reflecting the evolution of the information matrix, the above planning problem can be regarded as a finite horizon optimal control problem for a nonlinear system. It is thus a nonconvex problem and only suboptimal solutions can be guaranteed in practice. In this paper, it is shown that the problem (7) under reasonable assumptions can be reformulated and solved to global optimality.

\section{MODELING}

This section discusses the choice of objective function and assumptions regarding how the sensor can observe the targets, and presents the optimization problem solved in each iteration of the receding horizon control loop.

\section{A. Objective function}

1) Information measures: We seek to maximize the acquired information, which means that a scalar measure of the information matrix $\mathcal{I}$ is needed for the objective function in (7). The use of scalar measures of information matrices occurs also in the field of experiment design [17], e.g., in the $A$ - and $D$-optimality criteria. 
The most widely used scalarization is the $D$-optimality criterion, which seeks to maximize the determinant of the information matrix. This has a geometric interpretation as it corresponds to minimizing the volume of the resulting confidence ellipsoid. An equivalent convex criterion is to minimize

$$
l_{D}(\mathcal{I})=-(\operatorname{det} \mathcal{I})^{1 / n}
$$

where $n$ is the dimension of $\mathcal{I}$.

The $A$-optimality seeks to minimize the trace of the inverse of the information matrix,

$$
l_{A}(\mathcal{I})=\operatorname{tr}\left(\mathcal{I}^{-1}\right)
$$

which results in minimizing the total variance of the estimates. A semidefinite programming (SDP) formulation of the $A$-optimality criterion is obtained by introducing an auxiliary variable $T$ and minimizing $\operatorname{tr}(T)$ subject to

$$
T-\mathcal{I}^{-1} \succeq 0 \Longleftrightarrow\left[\begin{array}{ll}
T & I \\
I & \mathcal{I}
\end{array}\right] \succeq 0 .
$$

Several other scalarizations could be employed; [18] gives a thorough analysis of scalar performance measures for the Kalman filter and information filter.

2) Penalties on states and inputs: The objective function could also contain penalties on the state of the mobile sensor or the control input, e.g., to avoid large deviations from a pre-planned path or other unnecessary maneuvers.

3) Full objective function: For the remainder of the paper, a weighted $D$-optimality criterion is used for the information matrices. The weight parameters $\alpha_{k}^{i}$ are introduced to allow for prioritization of certain targets or promoting trajectories that acquire information at an early stage of the process. For the state of the mobile sensor and the control input, quadratic stage costs with weighting matrices $V_{x}$ and $V_{u}$ are used. Thus, for $M$ targets, the stage and terminal costs are given by

$$
\begin{aligned}
l_{k}\left(\mathcal{I}_{k}, x_{k}, u_{k}\right) & =u_{k}^{\top} V_{u} u_{k}+x_{k}^{\top} V_{x} x_{k}-\left(\operatorname{det} \mathcal{I}_{k}\right)^{1 / n}, \\
m\left(\mathcal{I}_{N}, x_{N}\right) & =x_{N}^{\top} V_{x} x_{N}-\left(\operatorname{det} \mathcal{I}_{N}\right)^{1 / n}
\end{aligned}
$$

where $\mathcal{I}_{k}=\operatorname{blkdiag}\left(\alpha_{k}^{1} \mathcal{I}_{k}^{1}, \ldots, \alpha_{k}^{M} \mathcal{I}_{k}^{M}\right)$ and $n$ is the size of $\mathcal{I}_{k}$. The above stage and terminal costs are separable in $\mathcal{I}, x$, and $u$, and yield a convex objective function.

\section{B. Information gain model}

The observation model (3) is assumed linear given that the target is within the FoV of the sensor, $\mathcal{V}_{k}$,

$$
y_{k}^{i}=H_{k} z_{k}^{i}+e_{k}^{i}
$$

where $e_{k}^{i}$ is Gaussian measurement noise, and that no information is obtained when it is not within the FoV. The discrete nature of the measurement model is modeled by introducing auxiliary binary variables $\delta_{k}^{i}$, which are nonlinear functions of $p^{i}$ and $x_{k}$, that represent if the target is within FoV and an observation is obtained or not. The sensor's FoV $\mathcal{V}_{k}=\left\{y \mid f_{\mathcal{V}}\left(x_{k}, y\right) \leq 0\right\}$ is a convex set, since $\left.f_{\mathcal{V}}\left(x_{k}, p\right)\right\}$ is assumed a convex function. Using this notation, and denoting the information gain from an observation $J_{k}=H_{k}^{\top} R_{k}^{-1} H_{k}$, (5) becomes

$$
\begin{aligned}
\mathcal{I}_{k+1} & =f_{\mathcal{I}}\left(\mathcal{I}_{k}, \delta_{k+1}\right) \\
& =\left(F_{k} \mathcal{I}_{k}^{-1} F_{k}^{\top}+G_{k} Q_{k} G_{k}^{\top}\right)^{-1}+\delta_{k+1} J_{k+1} .
\end{aligned}
$$

To simplify the optimization problem, $\delta_{k}^{i}$ will be treated as decision variables connected to the sensor state via constraints enforcing the FoV interpretation. Big- $M$ constraints [19],

$$
f_{\mathcal{V}}\left(x_{k}, p^{i}\right) \leq\left(1-\delta_{k}^{i}\right) M_{\delta},
$$

with $M_{\delta}$ large enough are used to realize this.

\section{Optimization problem}

With the information gain model and the objective function presented in the previous sections, the optimization problem that is solved in each sample of the receding horizon control loop is

$$
\begin{array}{ll}
\underset{x_{k}, u_{k}, \mathcal{I}_{k}, \delta_{k}}{\operatorname{minimize}} & \sum_{k=1}^{N-1} l_{k}\left(\mathcal{I}_{k}, x_{k}, u_{k}\right)+m\left(\mathcal{I}_{N}, x_{N}\right) \\
\text { subject to } & x_{k+1}=A_{k} x_{k}+B_{k} u_{k} \\
& \mathcal{I}_{k+1}^{i}=f_{\mathcal{I}}\left(\mathcal{I}_{k}^{i}, \delta_{k+1}^{i}\right) \\
& f_{\mathcal{V}}\left(x_{k}, p^{i}\right) \leq\left(1-\delta_{k}^{i}\right) M_{\delta} \\
& x_{k} \in \mathcal{X}, \quad u_{k} \in \mathcal{U}, \quad \delta_{k}^{i} \in\{0,1\}, \\
& x_{0}=\bar{x}_{0}, \quad \mathcal{I}_{0}^{i}=\overline{\mathcal{I}}_{0}^{i} .
\end{array}
$$

Even with a convex objective function, the problem (15) is an NP-hard mixed integer program and the fact that the problem has nonlinear equality constraints means that a straight-forward relaxation of the integer constraints results in a nonconvex problem. In the next section, we show that (15) can be reformulated into a problem that can be solved to global optimality using off-the-shelf solvers.

\section{Computing Globally Optimal Solutions}

This section derives a mixed integer semidefinite program that is equivalent to (15) and shows that this problem can be solved to global optimality using BnB.

A. Convex reformulation of the nonlinear equality constraints

The nonlinear equality constraints of (15) are nonconvex, as equality constraints in a convex optimization problem need to be affine [20]. Here, we show that they, in the problem of interest, can be relaxed to convex nonlinear inequality constraints and that the obtained problem is equivalent to the original nonconvex problem. The following definition will be needed:

Definition 1: (Matrix increasing, extension of [20].) Suppose $T \subseteq \mathbb{S}^{n}$ and let $\preceq$ denote the generalized inequality associated with the positive semidefinite cone. A function $f: \mathbb{S}^{n} \rightarrow \mathbb{S}^{m}$ is called matrix increasing on $T$ if

$$
x \preceq y, x \neq y \Longrightarrow f(x) \preceq f(y), f(x) \neq f(y),
$$


for all $x, y \in T$. For $m=1, f$ is called matrix increasing on $T$ if

$$
x \preceq y, x \neq y \Longrightarrow f(x)<f(y) .
$$

Matrix decreasing is defined analogously.

With this as background, the main result of the paper can now be formulated.

Theorem 1: Suppose that $f_{0}^{k}: \mathbb{S}^{n} \rightarrow \mathbb{R}, k=1, \ldots, N$ are matrix decreasing on $\mathbb{S}_{++}^{n}$, and that $f_{k}: \mathbb{S}^{n} \rightarrow \mathbb{S}^{n}, \quad k=0, \ldots, N-1, \quad$ are finite-valued functions that are matrix increasing on $\mathbb{S}_{++}^{n}$, and that $c \in \mathbb{S}_{++}^{n}$. Then, the optimal solution to the problem

$$
\begin{array}{ll}
\underset{x}{\operatorname{minimize}} & \sum_{k=1}^{N} f_{0}^{k}\left(x_{k}\right) \\
\text { subject to } & x_{k+1}=f_{k}\left(x_{k}\right), \quad k=0, \ldots, N-1 \\
& x_{0}=c,
\end{array}
$$

coincides with the optimal solution to the problem

$$
\begin{array}{ll}
\underset{x}{\operatorname{minimize}} & \sum_{k=1}^{N} f_{0}^{k}\left(x_{k}\right) \\
\text { subject to } & x_{k+1} \preceq f_{k}\left(x_{k}\right), \quad k=0, \ldots, N-1 \\
& x_{0} \preceq c .
\end{array}
$$

Proof: See Appendix I.

Consider a simplified version of the problem (15) with each of the binary variables $\delta_{k}^{i}$ given and fixed. This problem is then separable in $\mathcal{I}$ and $(x, u)$ and it is thus possible to optimize over $\mathcal{I}$ separately from $(x, u)$. When optimizing over $\mathcal{I}$, the relevant part of the objective function consists of the functions $f_{0}^{k}(\mathcal{I})=-(\operatorname{det} \mathcal{I})^{1 / n}$, which are matrix decreasing on $\mathbb{S}_{++}^{n}[20]$. Moreover, the information matrix recursion $f_{\mathcal{I}}(\cdot)$ has been shown to be matrix increasing [21], and given $\max \left|\operatorname{eig} J_{k}\right|<\infty$, it is also finite-valued. Hence, the simplified problem fulfills the requirements for Theorem 1, which means that the equality constraints representing the information matrix recursion can equivalently be replaced by inequality constraints.

Furthermore, since $Q_{k}$ is positive definite and $Q_{k}^{-1}$ thus exists, by applying the Woodbury matrix identity [22] and the Schur complement [20] to each of these inequalities, the inequality constraints involving both $\mathcal{I}$ and its inverse $\mathcal{I}^{-1}$ can be replaced by linear matrix inequalities involving $\mathcal{I}$ only:

$$
\begin{aligned}
& \mathcal{I}_{k+1}^{i} \preceq\left(F_{k} \mathcal{I}_{k}^{i-1} F_{k}^{\top}+G_{k} Q_{k} G_{k}^{\top}\right)^{-1}+\delta_{k+1}^{i} J_{k+1} \Longleftrightarrow \\
& \mathcal{I}_{k+1}^{i} \preceq F_{k}^{-\top} \mathcal{I}_{k}^{i} F_{k}^{-1}+\delta_{k+1}^{i} J_{k+1}-F_{k}^{-\top} \mathcal{I}_{k}^{i} F_{k}^{-1} G_{k} \times \\
& \\
& \times\left(Q_{k}^{-1}+G_{k}^{\top} F_{k}^{-\top} \mathcal{I}_{k}^{i} F_{k}^{-1} G_{k}\right)^{-1} G_{k}^{\top} F_{k}^{-\top} \mathcal{I}_{k}^{i} F_{k}^{-1} \\
& \quad \Longleftrightarrow \\
& 0 \preceq\left[\begin{array}{cc}
F_{k}^{-\top} \mathcal{I}_{k}^{i} F_{k}^{-1}-\mathcal{I}_{k+1}^{i}+\delta_{k+1}^{i} J_{k+1} & F_{k}^{-\top} \mathcal{I}_{k}^{i} F_{k}^{-1} G_{k} \\
G_{k}^{\top} F_{k}^{-\top} \mathcal{I}_{k}^{i} F_{k}^{-1} & Q_{k}^{-1}+G_{k}^{\top} F_{k}^{-\top} \mathcal{I}_{k}^{i} F_{k}^{-1} G_{k}
\end{array}\right] \\
& \triangleq \Gamma\left(\mathcal{I}_{k}^{i}, \mathcal{I}_{k+1}^{i}, \delta_{k+1}^{i}\right)
\end{aligned}
$$

By inserting this into (15), an equivalent problem is obtained:

$$
\begin{array}{ll}
\underset{x_{k}, u_{k}, \mathcal{I}_{k}, \delta_{k}}{\operatorname{minimize}} & \sum_{k=1}^{N-1} l_{k}\left(\mathcal{I}_{k}, x_{k}, u_{k}\right)+m\left(\mathcal{I}_{N}, x_{N}\right) \\
\text { subject to } & x_{k+1}=A_{k} x_{k}+B_{k} u_{k}, \\
& 0 \preceq \Gamma\left(\mathcal{I}_{k}^{i}, \mathcal{I}_{k+1}^{i}, \delta_{k+1}^{i}\right) \\
& f_{\mathcal{V}}\left(x_{k}, p^{i}\right) \leq\left(1-\delta_{k}^{i}\right) M_{\delta} \\
& x_{k} \in \mathcal{X}, \quad u_{k} \in \mathcal{U}, \quad \delta_{k}^{i} \in\{0,1\} \\
& x_{0} \preceq \bar{x}_{0}, \quad \mathcal{I}_{0}^{i} \preceq \overline{\mathcal{I}}_{0}^{i}
\end{array}
$$

which is a mixed integer semidefinite program. This means that the problem (15), for any given combination of the binary variables $\delta_{k}^{i}$ is equivalent to a convex SDP problem where the equality constraints representing the information matrix recursion are replaced by (18). Since the combination of binary variables is arbitrary, the reasoning holds for all sequences of $\delta$ and hence, the mixed integer problem in (15) can equivalently be reformulated using inequality constraints.

\section{B. Obtaining the solution using BnB}

In theory, the globally optimal solution to (19) could be obtained by solving the corresponding SDP problem for each of the possible combinations of the binary variables $\delta_{k}^{i}$, and pick the solution that results in the smallest objective value. However, as the number of possible combinations increases exponentially with the planning horizon $N$ and the number of targets $M$, this is likely to be computationally intractable.

In order to keep the computational complexity at a reasonable level, a $\mathrm{BnB}$ method is employed [15]. A prerequisite for the method to be meaningful is that convex relaxations are obtained when the binary constraints, $\delta_{k}^{i} \in\{0,1\}$, are relaxed to interval constraints, $\delta_{k}^{i} \in[0,1]$.

If the binary constraints in the original formulation (15) are relaxed to interval constraints, the obtained relaxation is a nonconvex problem and of little use in BnB. However, using Theorem 1, the original problem can be equivalently reformulated into a form (19) where the relaxations turn out to become convex problems, or more specifically, they turn out to be SDPs. This means that BnB can be used to efficiently solve (19) to global optimality, or alternatively to any user-defined certified level of suboptimality. Furthermore, the equivalence between the problems implies that their optimal solutions coincide, and that the globally optimal solution to (15) can be obtained by solving (19).

\section{EXPERIMENTS}

In this section, the performance of the proposed algorithm is compared to the performance of the RIGtree algorithm from [9]. The RIG-tree algorithm was implemented in Python while the optimization problems in our approach were solved in Matlab 2017b using YALMIP's BnB solver [23] which uses MOSEK [24] to solve the convex relaxations. All simulations were run on 


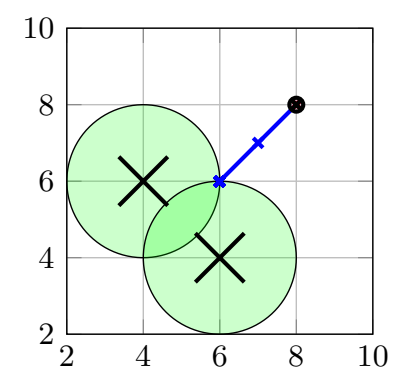

(a) Scenario 1

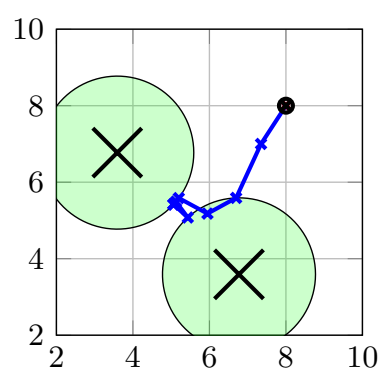

(b) Scenario 2
Fig. 1: Resulting trajectories for a mobile sensor starting at the black circle with the objective of maximizing the acquired information of the two targets located at the black crosses. The green regions are the areas from where the targets are visible.

a laptop with a $2.6 \mathrm{GHz}$ Intel i7 processor with $16 \mathrm{~GB}$ of RAM running Ubuntu 16.04.

Two scenarios, illustrated in Fig. 1, with two stationary targets are considered. The property of interest of both targets evolve according to a random walk model with $F_{k}=I$, where the process noise is ten times larger for target 1 (upper left) than for target $2: Q_{k}^{1}=10 \cdot Q_{k}^{2}=I$. The state of the mobile sensor represents its position and velocity, and the control input corresponds to its acceleration. A receding horizon approach with prediction horizon $N=10$ is employed, the sampling time is $T_{s}=1$, and the control input is limited to $u_{k} \in \mathcal{U}=\{u \mid-1 \leq u \leq 1\}$. The sensor's FoV is a circle centered in the sensor. Moreover, the information gained from a measurement is the identity matrix, $J_{k}=I$, the cost matrices are $V_{x}=\operatorname{diag}(0,0,0.1,0.1)$ and $V_{u}=0$, and the parameters $\alpha_{k}^{i}=1$. Hence, there is a small penalty on the velocity of the mobile sensor and no penalty on the control input.

Fig. 1 shows the optimized trajectories obtained using the mixed integer SDP formulation proposed in this paper. In Scenario 1, the sensor moves as fast as possible to a point from where both targets are visible, where it remains still. In Scenario 2, there is no such point. Instead, the sensor makes a quick visit to target 2 and then spends most time near the target with high process noise covariance.

Fig. 2 illustrates how the objective function of the first planning iteration improves as a function of the computation time for the proposed approach and the RIG-tree algorithm. Since the RIG-tree is a samplingbased method that returns different results each time it is called, and the performance in terms of objective value thus varies, Monte Carlo simulations are used to generate the relevant data.

BnB quickly finds a feasible solution which is nearly as good as the best solution found by the RIG-tree in all simulations and significantly better than the average solution. When given more time, $\mathrm{BnB}$ finds a better solution than the RIG-tree and also proves that it is the globally optimal solution. In contrast to our approach,

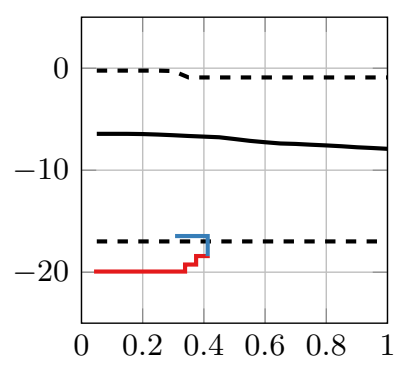

(a) Scenario 1

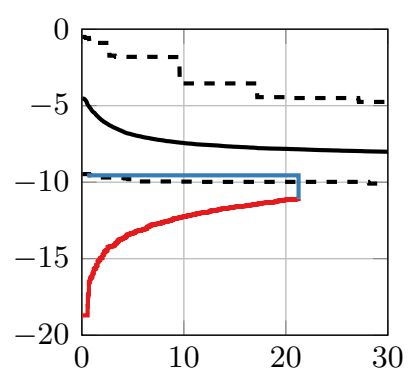

(b) Scenario 2
Fig. 2: Objective value as a function of computation time in seconds. Black: RIG-tree averaged over 1000 Monte Carlo runs. Black dashed: Worst and best case from RIGtree simulations. Red/blue: Lower/upper bound from BnB.

the RIG-tree does not provide a certificate regarding the level of suboptimality, which means that there is a risk of obtaining a poor solution (upper dashed line) without knowing that.

\section{Conclusions}

This work formulates an informative path planning problem as a receding horizon optimal control problem with nonlinear equality constraints, that at first glance seems intractable to solve to global optimality. However, the optimization problem is shown to be equivalent to a mixed integer SDP that robustly can be solved to global optimality using off-the-shelf optimization tools. Two illustrative simulations are provided to demonstrate that intuitively sound trajectories are computed. The proposed method provides global optimality guarantees and can be prematurely aborted once any user-defined level of suboptimality has been reached. These benefits have been confirmed in a numerical study.

\section{REFERENCES}

[1] A. Singh, A. Krause, C. Guestrin, W. J. Kaiser, and M. A. Batalin, "Efficient planning of informative paths for multiple robots." in Proceedings of AAAI International Joint Conference on Artificial Intelligence, Hyderabad, India, 2007, pp. 2204-2211.

[2] G. W. Ng and K. H. Ng, "Sensor management - what, why and how," Information Fusion, vol. 1, no. 2, pp. $67-75,2000$.

[3] S. Joshi and S. Boyd, "Sensor selection via convex optimization," IEEE Trans. Signal Processing, vol. 57, no. 2, pp. 451$462,2009$.

[4] H. Wang, K. Yao, G. Pottie, and D. Estrin, "Entropy-based sensor selection heuristic for target localization," in Proceedings of the 3rd international symposium on Information processing in sensor networks, Berkeley, CA, USA, 2004, pp. 3645.

[5] M. P. Vitus, W. Zhang, A. Abate, J. Hu, and C. J. Tomlin, "On efficient sensor scheduling for linear dynamical systems," Automatica, vol. 48, no. 10, pp. 2482-2493, 2012.

[6] R. Takei, R. Tsai, Z. Zhou, and Y. Landa, "An efficient algorithm for a visibility-based surveillance-evasion game," Communications in Mathematical Sciences, vol. 12, no. 7, pp. 1303-1327, 2014.

[7] P. Skoglar, "Tracking and planning for surveillance applications," Ph.D. dissertation, Department of Electrical Engineering, Linköping University, 2012.

[8] S. Patil, G. Kahn, M. Laskey, J. Schulman, K. Goldberg, and P. Abbeel, "Scaling up gaussian belief space planning 
through covariance-free trajectory optimization and automatic differentiation," in Algorithmic Foundations of Robotics XI. Springer, 2015, pp. 515-533.

[9] G. A. Hollinger and G. S. Sukhatme, "Sampling-based robotic information gathering algorithms," The International Journal of Robotics Research, vol. 33, no. 9, pp. 1271-1287, 2014.

[10] G. M. Hoffmann and C. J. Tomlin, "Mobile sensor network control using mutual information methods and particle filters," IEEE Trans. Automat. Contr., vol. 55, no. 1, pp. 32-47, 2010.

[11] B. J. Julian, M. Angermann, M. Schwager, and D. Rus, "Distributed robotic sensor networks: An information-theoretic approach," The International Journal of Robotics Research, vol. 31, no. 10, pp. 1134-1154, 2012.

[12] S. S. Ponda, R. M. Kolacinski, and E. Frazzoli, "Trajectory optimization for target localization using small unmanned aerial vehicles," in Proceedings of AIAA Guidance, Navigation, and Control Conference, Chicago, IL, USA, 2009, pp. 1209-1220.

[13] J. Le Ny and G. J. Pappas, "On trajectory optimization for active sensing in gaussian process models," in Proceedings of the 48th IEEE Conference on Decision and Control, Shanghai, China, 2009, pp. 6286-6292.

[14] N. Atanasov, J. Le Ny, K. Daniilidis, and G. J. Pappas, "Information acquisition with sensing robots: Algorithms and error bounds," in Proceedings of IEEE International Conference on Robotics and Automation, Hong Kong, China, 2014, pp. 64476454 .

[15] C. A. Floudas, Nonlinear and mixed-integer optimization: Fundamentals and applications. London, UK: Oxford University Press, 1995.

[16] T. Kailath, A. Sayed, and B. Hassibi, Linear Estimation. Upper Saddle River, NJ, USA: Prentice Hall, 2000.

[17] F. Pukelsheim, Optimal design of experiments. New York, USA: John Wiley \& Sons, 1993.

[18] C. Yang, L. Kaplan, and E. Blasch, "Performance measures of covariance and information matrices in resource management for target state estimation," IEEE Trans. Aerosp. Electron. Syst., vol. 48, no. 3, pp. 2594-2613, 2012.

[19] H. P. Williams, Model building in mathematical programming. New York, USA: John Wiley \& Sons, 2013

[20] S. Boyd and L. Vandenberghe, Convex Optimization. Cambridge, UK: Cambridge University Press, 2004

[21] G. Hendeby and F. Gustafsson, "Fundamental filtering limitations in linear non-Gaussian systems," in Proceedings of the 16th Triennial IFAC World Congress, Prague, Czech Republic, 2005 , pp. $1209-1220$.

[22] G. H. Golub and C. F. Van Loan, Matrix Computations (3rd Ed.). Baltimore, MD, USA: Johns Hopkins University Press, 1996.

[23] J. Löfberg, "YALMIP : A toolbox for modeling and optimization in MATLAB," in Proceedings of the CACSD Conference, Taipei, Taiwan, 2004

[24] MOSEK ApS, The MOSEK optimization toolbox for MATLAB manual. Version 8.1., 2017.

\section{APPENDIX I \\ Proof of Theorem 1}

Note that a problem equivalent to (17) can be obtained by introducing slack variables $\varepsilon_{0}, \ldots, \varepsilon_{N}$ and replacing each inequality constraint in (17) with an equality constraint and a positive semidefiniteness constraint. This yields the following equivalent problem

$$
\begin{array}{ll}
\underset{x, \varepsilon}{\operatorname{minimize}} & \sum_{k=1}^{N} f_{0}^{k}\left(x_{k}\right) \\
\text { subject to } & x_{k+1}+\varepsilon_{k+1}=f_{k}\left(x_{k}\right), \quad k=0, \ldots, N-1 \\
& x_{0}+\varepsilon_{0}=c, \\
& \varepsilon_{i} \succeq 0, \quad k=0, \ldots, N .
\end{array}
$$

We will now show that the optimal choice of the slack variables in $(20)$ is $\varepsilon_{k}=0, k=0, \ldots, N$ and that the inequality constraints of (17) are satisfied with equality at optimality. We begin by optimizing over some of the variables, namely the final variables $x_{N}$ and $\varepsilon_{N}$. To do this, we introduce the function $f_{0}: \mathbb{S}^{n} \rightarrow \mathbb{R}$, which we assume is matrix decreasing on $\mathbb{S}_{++}^{n}$, and make use of the following help problem

$$
\begin{array}{ll}
\underset{x, \varepsilon}{\operatorname{minimize}} & f_{0}(x) \\
\text { subject to } & x+\varepsilon=c_{0}, \\
& \varepsilon \succeq 0,
\end{array}
$$

which can be considered as a parametric program of the parameter $c_{0}$. Since the objective function is matrix decreasing,

$$
x \preceq x+\varepsilon, x \neq x+\varepsilon \Longrightarrow f_{0}(x)>f_{0}(x+\varepsilon),
$$

a nonzero slack $\varepsilon$ implies a suboptimal $x$. Hence, for any finite choice of $c_{0}$, the unique optimal solution to (21) is given by $x^{\star}=c_{0}, \varepsilon^{\star}=0$. In particular, this holds for $c_{0}=f_{k}\left(x_{k}\right)$, which means that the variables $\varepsilon_{N}=0$, $x_{N}=f_{N-1}\left(x_{N-1}\right)$ can be eliminated together with the corresponding equality constraint in (20).

After the elimination of the variables in stage $N$, the problem in (20) can be reduced to

$$
\begin{array}{ll}
\underset{x, \varepsilon}{\operatorname{minimize}} & \sum_{k=1}^{N-1} f_{0}^{k}\left(x_{k}\right)+f_{0}^{N}\left(f_{N}\left(x_{N-1}\right)\right) \\
\text { subject to } & x_{k+1}+\varepsilon_{k+1}=f_{k}\left(x_{k}\right), \quad k=0, \ldots, N-2 \\
& x_{0}+\varepsilon_{0}=c, \\
& \varepsilon_{k} \succeq 0 . \quad k=0, \ldots, N-1,
\end{array}
$$

which has the same structure as the original problem (20); the second term of the objective function is matrix decreasing, as it is a matrix decreasing function of a matrix increasing function, the constraint functions are matrix increasing and finite-valued, and $c \in \mathbb{S}_{++}^{n}$.

The procedure of optimizing over, and eliminating, the final variables can thus be applied repeatedly until all variables except $x_{0}$ have been eliminated, which eventually gives an equivalent problem

$$
\begin{array}{ll}
\underset{x, \varepsilon}{\operatorname{minimize}} & \sum_{k=1}^{N} f_{0}^{k}\left(f_{k}\left(f_{k-1}\left(\ldots f_{0}\left(x_{0}\right)\right)\right)\right) \\
\text { subject to } & x_{0}=c,
\end{array}
$$

which has the trivial unique and finite solution $x_{0}=c$. This means that the unique optimal solution to (20) and thus also (17) is given by $x_{0}^{\star}=c, x_{k+1}^{\star}=f_{k}\left(x_{k}^{\star}\right), \varepsilon_{k}^{\star}=$ $0, k=0, \ldots, N$, and that $x_{k}$, by induction, is finite for all $k$.

Note that the constraints of (17) are relaxations of the constraints of (16). Hence, the feasible set for (16) is a subset of the feasible set for (17) and the optimal value for (16) can thus not be lower than the optimal value for (17). Since $x_{0}^{\star}=c, x_{k+1}^{\star}=f_{k}\left(x_{k}^{\star}\right), k=0, \ldots, N-1$, is an optimal solution to (17) and feasible for (16), it must also be an optimal solution to (16). Hence, the optimal solution to (17) coincides with the optimal solution to (16), which concludes the proof. 